\title{
IDEALS CONTAINING MONICS
}

\author{
BUDH NASHIER AND WARREN NICHOLS
}

\begin{abstract}
If an ideal $I$ of $R[X]$ contains a monic, then every monic in $I$ modulo $J R[X]$ ( $J$ being an ideal contained in the Jacobson radical of $R$ ) can be lifted to a monic in $I$. This result is used to give an elementary proof of Horrocks' Theorem.
\end{abstract}

1. Introduction. Let $R$ be a ring, $J$ an ideal contained in the Jacobson radical of $R$, and let $X$ denote an indeterminate. In this note we show that if a left ideal $I$ of $R[X]$ contains a monic, then every monic in $(I+J R[X]) / J R[X]$ can be lifted to a monic in $I$. Using this, we show that when $R$ is commutative and quasi-local, an invertible ideal $I$ of $R[X]$ which contains a monic is principal. This is the "rank one" case of Horrocks' Theorem: If $P$ is a projective $R[X]$-module which becomes free upon inversion of a monic, then $P$ is free. We show that Horrocks' Theorem follows by induction, thus providing the simplest proof which has appeared to date of that crucial component of Quillen's solution to the Serre Conjecture.

2. Lifting monics. Let $R$ be a ring, $J$ an ideal contained in the Jacobson radical of $R, \bar{R}=R / J$. We shall identify $R[X] / J R[X]$ with $\bar{R}[X]$; thus, for $g \in$ $R[X]$, we say that $g+J R[X]$ is a monic of degree $n$ in $\bar{R}[X]$ if the coefficient of $X^{n}$ in $g$ is 1 and the coefficients of higher powers of $X$ lie in $J$.

THEOREM 1. Let $I$ be a left ideal of $R[X]$ which contains a monic. Then every monic in $(I+J R[X]) / J R[X]$ is of the form $g+J R[X]$ for some monic $g$ in $I$.

PROOF. Let $\alpha$ be a monic in $(I+J R[X]) / J R[X]$ of degree $r$. We first suppose that $I$ contains a monic $f$ of degree $r+1$. Write $\alpha=g_{1}+J R[X], g_{1} \in I$; then $g_{1}$ will have degree $m \geq r$. Let $a$ be the coefficient of $X^{m}$ in $g_{1}$. If $m=r$, then $1-a \in J$, so $a$ is a unit of $R$ and $g=a^{-1} g_{1}$ is our required monic. If $m>r$, then $a \in J$. Set $g_{2}=g_{1}-a X^{m-r-1} f \in I$. Then $g_{2}+J R[X]=g_{1}+J R[X]=\alpha$ and degree $g_{2}<$ degree $g_{1}$. We continue until we obtain the required $g$.

Next we show that $I$ actually contains a monic of degree $r+1$. We know that $I$ contains a monic, and that an ideal containing a monic of degree $d$ will contain a monic of each higher degree. It suffices to show that if $I$ contains a monic of degree $n>r+1$, then $I$ contains a monic of degree $n-1$. Since $n-1 \geq r$, $(I+J R[X]) / J R[X]$ contains a monic $\beta$ of degree $n-1$. By the above, there is a monic $h$ in $I$ (necessarily of degree $n-1$ ) with $h+J R[X]=\beta$, and we are done.

Received by the editors May 10, 1985 and, in revised form, February 3, 1986.

1980 Mathematics Subject Classification (1985 Revision). Primary 13C10. 
3. Invertible ideals containing monics. From now on, $R$ will denote a commutative ring with unique maximal ideal $\mathfrak{m}$. We write $R / \mathfrak{m}=k$, the residue field.

THEOREM 2 (cf. [Lam, IV, Theorem 4.1, case $n=1]$ ). Let I be an invertible ideal of $R[X]$ containing a monic. Then $I=f R[X]$ for some monic $f \in I$.

Proof. Note that $(I+\mathfrak{m} R[X]) / \mathfrak{m} R[X]$ is a nonzero ideal of $k[X]$, a principal ideal domain. Thus $(I+\mathfrak{m} R[X]) / \mathfrak{m} R[X]=\alpha k[X]$, where $\alpha \in k[X]$ may be taken to be monic. By Theorem 1 , we can find $f \in I$ monic such that $f+\mathfrak{m} R[X]=\alpha$. Then $I=f R[X]+I \cap \mathfrak{m} R[X]$. We assert that $I \cap \mathfrak{m} R[X]=\mathfrak{m} I$. To see this, write $I \cap \mathfrak{m} R[X]=I L$, where $L=I^{-1}(I \cap \mathfrak{m} R[X])$ is an ideal of $R[X]$. We have $I L \subseteq \mathfrak{m} R[X]$, but $I \nsubseteq \mathfrak{m} R[X]$ (since $I$ contains a monic). Thus $L \subseteq \mathfrak{m} R[X]$, and so $I \cap \mathfrak{m} R[X] \subseteq \mathfrak{m} I$. The reverse containment is clear, so the assertion follows. Thus we have $I=f R[X]+\mathfrak{m} I$, with $f \in I$ monic. Write $M=I / f R[X]$. Since invertible ideals are finitely generated, $M$ is finitely generated. As $f M=(0), M$ is a finitely generated $R[X] / f R[X]$ module; as $f$ is monic, $M$ is a finitely generated $R$-module. Since $\mathfrak{m} M=M, M$ must be zero by Nakayama's Lemma. Thus $I=f R[X]$, as required.

4. Horrocks' Theorem. As above, $R$ denotes a commutative ring with unique maximal ideal $\mathfrak{m}, k=R / \mathfrak{m}$ the residue field. We write $A=R[X], S$ for the set of monics of $A$, and "bar" for the functor $\bigotimes_{R} k$.

HoRRoCKS' THEOREM. Let $P$ be a finitely generated projective A-module. If $P_{S}$ is a free $A_{S}$-module, then $P$ is a free $A$-module.

Proof. Since $A$ has no nontrivial idempotents, $P$ has a rank; say rank $P=n$. We proceed by induction on $n$.

When $n=1, P$ is isomorphic to an ivertible ideal of $A$ which contains a monic; indeed, if $\phi: P \rightarrow A$ is an $A$-module map for which $\phi_{S}$ is an isomorphism, then $I=\phi(P)$ is the required ideal. By Theorem 2, $P$ is free.

Now suppose $n \geq 2$. Let $p_{1}, \ldots, p_{n} \in P$ be an $A_{S}$-basis of $P_{S}$. We have that $\bar{P}$ is a free $k[X]$-module of rank $n$. Using (for example) the normal form theorem for $m \times n$ matrices over a principal ideal domain (with $m=1$ ), we can find a basis $\bar{q}_{1}, \ldots, \bar{q}_{n}$ of $\bar{P}$ for which $\bar{p}_{1}=\alpha \bar{q}_{2}$, for some $\alpha \in k[X]$. Making use of an idea from [Roitman, Lemma 10] we set $p=q_{1}+X^{t} p_{1}$, with $t$ to be specified later. Then $\bar{p}=\bar{q}_{1}+X^{t} \alpha \bar{q}_{2}$, so $\bar{p}, \bar{q}_{2}, \ldots, \bar{q}_{n}$ is a basis of $\bar{P}$. We have $s q_{1}=\sum_{i=1}^{n} a_{i} p_{i}$ for some $s \in S, a_{1}, \ldots, a_{n} \in A$. Thus $s p=\left(a_{1}+s X^{t}\right) p_{1}+\sum_{i=2}^{n} a_{i} p_{i}$. Select $t$ large enough so that $a_{1}+s X^{t}$ is monic. Then $p, p_{2}, \ldots, p_{n}$ is an $A_{S}$-basis of $P_{S}$.

Write $T=1+\mathfrak{m} R[X]$. Then $\mathfrak{m}$ is contained in the Jacobson radical of $A_{T}$. Since $\bar{p}, \bar{q}_{2}, \ldots, \bar{q}_{n}$ span $\bar{P}_{T}=\bar{P}$, it follows that $p, q_{2}, \ldots, q_{n}$ span $P_{T}$. Since $P_{T}$ is projective of rank $n$, we conclude that $P_{T}$ is actually free, with basis $p, q_{2}, \ldots, q_{n}$.

Now consider $P^{\prime}=P / A p$. Note that $P_{S}^{\prime}$ and $P_{T}^{\prime}$ are free of rank $n-1$. Any maximal ideal $\mathfrak{M}$ of $A$ either avoids $S$ or avoids $T$, since if $\mathfrak{M}$ meets $S$ the field $A / \mathfrak{M}$ is an integral extension of the domain $R /(R \cap \mathfrak{M})$, which thus must be a field. Thus $P_{\mathfrak{M}}^{\prime}$ is free of rank $n-1$, and hence [Bourbaki, Chapter II, $\S 5.2$, Theorem 1] $P^{\prime}$ is projective. By induction, $P^{\prime}$ is free. Then $P \cong A p \oplus P^{\prime}$ is free, and we are done. 


\section{REFERENCES}

[Bourbaki] N. Bourbaki, Commutative algebra, Addison-Wesley, 1972.

[Lam] T. Y. Lam, Serre's Conjecture, Lecture Notes in Math., vol. 635, Springer-Verlag, Berlin and New York, 1978.

[Roitman] Moshe Roitman, On projective modules over polynomial rings, J. Algebra 58 (1979), 51-63.

Department of MAThematics, Florida State University, Tallahassee, FLORIDA 32306-3027 